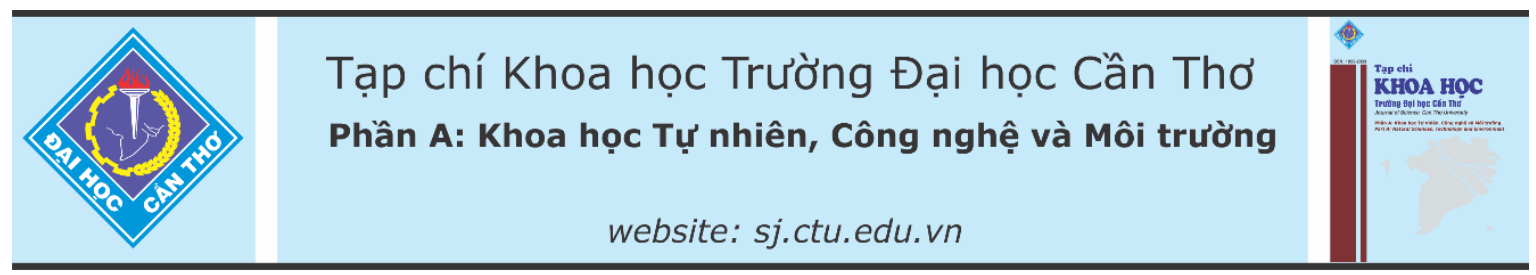

DOI:10.22144/ctu.jvn.2021.144

\title{
TÍNH CHẤT VẬN CHUYỂN CỦA KHÍ ĐIỆN TỬ HAI CHIỂU TRONG GIẾNG THẾ SiGe/Si/SiGe DƯới ẢNH HƯởNG CỦA NHIỆT Độ VÀ TÙ TRƯờnG
}

\author{
Nguyễn Thị Thúy Quỳnh ${ }^{1^{*}}$, Lý Hoàng Diễm ${ }^{1}$, Nguyễn Thị Quế Trinh ${ }^{1}$ và Phạm Tiến Phát ${ }^{2}$ \\ ${ }^{1}$ Khoa Su phạm và Xã hội Nhân văn, Truòng Đại học Kiên Giang \\ ${ }^{2}$ Cựu học viên Trường Đại học Khoa học Tự nhiên, Đại học Quốc gia Thành phố Hồ Chí Minh \\ *Người chịu trách nhiệm về bài viết: Nguyễn Thị Thúy Quỳnh (email: nttquynh@vnkgu.edu.vn)
}

\section{Thông tin chung:}

Ngày nhận bài: $19 / 05 / 2021$

Ngày nhận bài sủa: 21/07/2021

Ngày duyệt đăng: 29/10/2021

Title:

Transport properties of twodimensional electron gas in $\mathrm{SiGe/Si/SiGe} \mathrm{quantum} \mathrm{well}$ under effects of temperature and in-plane applied magnetic field

\section{Tù khóa:}

Chuyển pha kim loai-cách điện, giếng thế, khi điện tử, $\mathrm{Si} / \mathrm{SiGe}$, tán xa giao diện nhám, tán xa tạp chất xa

\section{Keywords:}

Electron gas, interfaceroughness scattering, metalinsulator transition, quantum well, remote charged-impurity scattering, Si/SiGe

\begin{abstract}
The mobility of two-dimensional electron gas in SiGe/Si/SiGe quantum well at finite temperatures is investigated for two cases: with and without in-plane magnetic field, considering two scattering mechanisms: remote charged-impurity and interface-roughness scattering. Exchangecorrelation effects and multiple-scattering effects are also taken into account. The dependence of critical density of the metal-insulator transition on the carrier density, layer thickness, remote doping distance, temperature and magnetic field are studied. For temperatures lesser than $2 \mathrm{~K}$, the calculation is in good agreement with previous results. The results can be used as guideline to grow Sillicon samples and control the temperature of system when measuring critical density and obtain information about the scattering mechanisms in the SiGe/Si/SiGe quantum well.
\end{abstract}

\section{TÓM TẮT}

Nghiên cúu đuoợc thưc hiện nhằm khảo sát độ linh động của khi điện tư hai chiều trong một giếng luợng tư SiGe/Si/SiGe tại nhiệt độ bất kỳ khi không có tù truờng và khi bị phân cực bời tù truờng, xem xét tới hai co chế tán xạ: tán xạ tạp chất xa và tán xạ giao diện nhám có tính tới hiệu ưng twơng quan-trao đổi và hiệu chỉnh truờng cuc bộ. Bên cạnh đó, sụ phu thuộc của mật độ tới hạn vào mạt độ hạt tải, bề rộng giếng thế, khoảng cách lớp tạp chất, nhiệt độ và tù trường cũng được nghiên cưu. Tại nhiệt độ duới $2 K$, kết quả nghiên cúu này phù hợp với các kết quả đỉ truơóc. Các kết quả này có thể sư dụng để định hướng thực nghiệm trong viẹcc nuôi cấy mẫu và kiểm soát nhiệt độ của hệ khi đo đạc mật độ tới hạn và thông tin về các co chế tán xa trong giếng luợng tử SiGe/Si/SiGe.

\section{GIỚI THIÊU}

Khi tính đến các hiệu ứng tương quan-trao đổi (ECE) và đa tán xạ (MSE), các tính toán cho độ linh động của khí điện tử hai chiều (2DEG) trong các cấu trúc điện tử khác nhau với các cơ chế tán xạ khác nhau có thể giải thích được sự xuất hiện của chuyển pha kim loại-cách điện (MIT) ở các mật độ hạt tải thấp với các kết quả phù hợp tốt với thực nghiệm (Gold, 2010, 2011). Bằng hướng tiếp cận đó, Gold (2010) cho thấy tán xạ tạp chất xa (RIS) là cơ chế chủ yếu gây ra MIT trong một mẫu giếng thế lượng tử $(\mathrm{QW}) \mathrm{SiGe} / \mathrm{Si} / \mathrm{SiGe}$ trong số liệu thực nghiệm của Wilamowski et al. (2001) và đã có nhận định sơ bộ rằng tác dụng của tán xạ giao diện nhám (IRS) 
chỉ trở nên đáng kể trong những $\mathrm{QW}$ có bề rộng hẹp hơn, tức là bằng việc thay đổi bề rộng $\mathrm{QW}$, có thể tăng cường hoặc giảm nhẹ tác dụng của IRS lên MIT.

Trong nghiên cứu này, định lượng sự phụ thuộc của MIT vào bề rộng $\mathrm{QW}$ được khảo sát nhằm xác nhận rằng những $\mathrm{QW}$ hẹp rất phù hợp để nghiên cứu MIT gây ra do cơ chế IRS vì tác dụng của cơ chế tán xạ này trở nên rõ rệt. Hơn nữa, mô hình được sử dụng có thể tiếp cận các giá trị nhiệt độ tiệm cận 0 $\mathrm{K}$, nhờ đó có thể đánh giá tính hợp lý trong cách tiếp cận của Gold (2010) khi tác giả này dùng một mô hình tại 0 độ tuyệt đối để đối chiếu với số liệu thực nghiệm được đo ở nhiệt độ $0,36 \mathrm{~K}$. Qua đó, các giới hạn mật độ - nhiệt độ của mô hình được đề xuất mà ở đó kết quả tính toán là đáng tin cậy.

Bên cạnh đó, nghiên cứu chỉ ra tác dụng của nhiệt độ ở các giá trị dưới $40 \mathrm{~K}$ lên độ linh động và mật độ tới hạn là đáng kể thậm chí khi tương tác phonon-hạt tải yếu thông qua ảnh hưởng lên hiệu ứng chắn. Các kết quả trong nghiên cứu còn được mở rộng cho trường hợp $2 \mathrm{DEG}$ bị phân cực bằng một từ trường có giá trị bão hòa.

\section{CƠ SỞ LÝ THUYÊT}

\subsection{Mô hình giếng lượng tử hình chữ nhật}

Điện tử trong lớp $\mathrm{Si}$ của cấu trúc $\mathrm{SiGe} / \mathrm{Si} / \mathrm{SiGe}$ có thể xem là một $2 \mathrm{DEG}$ bên trong một $\mathrm{QW}$ hình chữ nhật. Do đó, các trạng thái của điện tử bị lượng tử hóa thành các dải con. Với mật độ hạt tải nhỏ hơn $30 \times 10^{11} \mathrm{~cm}^{-2}$, các tính toán của Zeller and Abstreiter (1986) cho thấy chỉ dải con dưới cùng bị chiếm đóng. Theo Gold (1987), dải con này có quy luật tán sắc parabolic và khối lượng hiệu dụng $m^{*}=0,19 m_{e}$ theo phương giao diện $\mathrm{SiGe/Si} \mathrm{(mặt} \mathrm{phẳng} x y$ vuông góc với phương nuôi $z$ ), còn khối lượng hiệu dụng theo phương $z$ là $m_{z}=0,916 m_{e}$ với $m_{e}$ là khối lượng của điện tử tự do.

Các ước lượng cho mức Fermi tại mật độ $10 \times 10^{11} \mathrm{~cm}^{2}$ và bề rộng giếng thế $3 \mathrm{~nm}, 10 \mathrm{~nm}, 20$ nm lần lượt là $48,0 \mathrm{meV}, 6,7 \mathrm{meV}$ và $3,6 \mathrm{meV}$ trong khi chiều cao rào thế khoảng $370 \mathrm{meV}$. Năng lượng nhiệt của điện tử tại nhiệt độ $40 \mathrm{~K}$ vào cỡ $3,4 \mathrm{meV}$ - đủ bé để điện tử từ dải con thấp nhất khó có thể nhảy lên các dải con phía trên (Gold, 1987). Do đó, mô hình giếng thế sâu vô hạn được sử dụng. Hơn nữa tỉ số $\left(\mathrm{m}^{*} / \mathrm{m}_{\mathrm{z}}\right)^{2}=0,04$ đủ bé để bỏ qua hiệu ứng xuyên rào của điện tử từ bên trong $\mathrm{QW}$ vào lớp $\mathrm{SiGe}$ (Laikhtman \& Kiehl, 1993). Khi đó, hàm sóng của điện tử theo phương $z$ có dạng

$$
\psi(z)= \begin{cases}\sqrt{\frac{2}{a}} \sin \left(\frac{\pi z}{a}\right), & 0<z<a, \\ 0, & z \leq 0 \text { hay } z \geq a .\end{cases}
$$

trong đó $a$ là bề rộng của $\mathrm{QW}$.

\section{2. Độ linh động của điện tử trong mô hình một dải con}

Khi từ trường $B$ song song với phương $x y$ được áp vào hệ, mật độ hạt tải $n_{ \pm}$đối với spin lên/xuống không bằng nhau. Gọi $n=n_{+}+n_{-}$là tổng mật độ hạt tải và $B_{s}$ là giá trị trường bão hòa được cho bởi $g \mu_{B} B_{s}=2 E_{F}$, trong đó $g$ là hệ số spin của điện tử, $\mu_{B}$ là Bohr magneton và $E_{F}$ là năng lượng Fermi. Đối với $T>0, n_{ \pm}$được xác định từ (Das Sarma \& Hwang, 2005)

$$
\begin{aligned}
& n_{+}=\frac{n}{2} t \ln \frac{1-e^{2 b / t}+\sqrt{\left(e^{2 b / t}-1\right)^{2}+4 e^{2(b+1) / t}}}{2}, \\
& n_{-}=n-n_{+},
\end{aligned}
$$

trong đó $b=B / B_{s}, t=T / T_{F}$ với $T_{F}$ là nhiệt độ Fermi. Khi $t$ đủ gần 0 , hệ phương trình (2) tiến về dạng

$$
\begin{aligned}
& n_{ \pm}=\frac{n}{2}\left(1 \pm \frac{B}{B_{s}}\right), B<B_{s} \\
& n_{+}=n, n_{-}=0, B \geq B_{s} .
\end{aligned}
$$

Trong gần đúng thời gian hồi phục, biểu thức tính thời gian hồi phục trung bình cho mỗi thành phần \pm được cho bởi lý thuyết Drude-Boltzmann nhiệt độ hữu hạn

$$
\left\langle\tau_{ \pm}\right\rangle=\frac{\int-\frac{\partial f^{ \pm}(\varepsilon)}{\partial \varepsilon} \tau(\varepsilon) \varepsilon d \varepsilon}{\int-\frac{\partial f^{ \pm}(\varepsilon)}{\partial \varepsilon} \varepsilon d \varepsilon}
$$

$$
\text { trong đó } f^{ \pm}(\varepsilon)=\frac{1}{1+\exp \left[\beta \varepsilon-\beta \mu^{ \pm}(T)\right]} \text { là hàm }
$$

Fermi, $\quad \beta=\left(k_{B} T\right)^{-1}, \quad \varepsilon=\hbar^{2} k^{2} /\left(2 m^{*}\right) \quad$ và $\mu^{ \pm}=\ln \left[\exp \left(\beta E_{F}^{ \pm}\right)-1\right] / \beta . \quad E_{F}^{ \pm}$là năng lượng Fermi cho mỗi thành phần của spin tìm từ 
$E_{F}^{ \pm}=\frac{\hbar^{2}\left(k_{F}^{ \pm}\right)^{2}}{2 m^{*}}, k_{F}=\sqrt{2 \pi n / g_{v}}, k_{F}^{ \pm}=\sqrt{4 \pi n_{ \pm} / g_{v}}$

trong đó $g_{v}=2$ là suy biến valley của hệ.

$\tau(\varepsilon)$ trong phương trình (4) là thời gian hồi phục theo năng lượng, $\tau_{k}$ là biểu diễn của nó theo số sóng. Các lý luận ở phần 2.1 cho thấy điện tử chỉ chiếm dải con thấp nhất nên chỉ các cơ chế tán xạ bên trong dải con này được quan tâm. Hơn nữa, Laikhtman and Kiehl (1993) cho thấy tương tác spin-quỹ đạo cũng có thể bỏ qua vì khoảng phân tách giữa các trạng thái do hiệu ứng này chỉ cỡ 40 meV. Khi đó, $\tau_{k}$ cho bởi lý thuyết vận chuyển tuyến tính có dạng

$$
\frac{1}{\tau_{k}}=\frac{1}{2 \pi \hbar \varepsilon} \int_{0}^{2 k} \frac{\left\langle|U(q)|^{2}\right\rangle}{\epsilon_{q}^{2}} \frac{q^{2} d q}{\sqrt{4 k^{2}-q^{2}}}
$$

trong đó $\in_{q}$ là hàm điện môi đặc trưng cho hiệu ứng chắn. Trong xấp xỉ pha ngẫu nhiên, nó có dạng

$$
\in_{q}=1+V(q) F_{C}(q) \Pi(q, T)[1-G(q)] .
$$

Trong phương trình $(6), V(q)$ là ảnh Fourier của thế Coulomb, $V(q)=2 \pi e^{2} /\left(\varepsilon_{L} q\right), \quad \varepsilon_{L}=12,5$ là hằng số điện môi của hệ được khảo sát. $F_{C}(q)$ là thừa số dạng khi tính tới hiệu ứng bề rộng khác 0 của lớp 2DEG trong QW, có dạng (Gold, 1987)

$$
F_{C}(q)=\frac{1}{4 \pi^{2}+a^{2} q^{2}}\left(3 a q+\frac{8 \pi^{2}}{a q}-\frac{32 \pi^{4}}{a^{2} q^{2}} \frac{1-e^{a q}}{4 \pi^{2}+a^{2} q^{2}}\right) .
$$

Ngoài ra, để hàm điện môi ở phương trình (6) có thể mô tả tốt ảnh hưởng của thế tương quan-trao đổi ở mật độ thấp, một dạng của hiệu chỉnh trường cục bộ (LFC) $G(q)$ được cho trong Gold (1997) được sử dụng. Hàm phân cực bất khả quy cho 2DEG ở nhiệt độ hữu hạn là tổng $\Pi(q, T)=\Pi_{+}(q, T)+\Pi_{-}(q, T)$ với mỗi thành phần của spin được xác định từ (Das Sarma \& Hwang, 2005)

$$
\Pi_{ \pm}(q, T)=\frac{\beta}{4} \int_{0}^{\infty} d \mu^{\prime} \frac{\Pi_{ \pm}^{0}\left(q, \mu^{\prime}\right)}{\cosh ^{2} \beta\left(\mu^{ \pm}-\mu^{\prime}\right) / 2} .
$$

$\Pi_{ \pm}^{0}\left(q, \mu^{\prime}\right)$ là hàm phân cực ở nhiệt độ $T=0$, cho bởi
$\Pi_{ \pm}^{0}\left(q, E_{F}^{ \pm}\right)=\frac{g_{v} m^{*}}{2 \pi \hbar^{2}}\left[1-\sqrt{1-\left(\frac{2 k_{F}^{ \pm}}{q}\right)^{2}} \theta\left(q-2 k_{F}^{ \pm}\right)\right]$

với $\theta(x)$ là hàm bước.

Phương trình (5) cho thấy các cơ chế tán xạ ảnh hưởng tới thời gian hồi phục $\tau_{k}$ thông qua đại lượng $\left\langle|U(q)|^{2}\right\rangle$ là hàm tự tương quan ( $\left.\mathrm{ACF}\right)$ của thế tán xạ trong không gian vector sóng. Khi các cơ chế tán xạ là yếu và độc lập nhau, $\left\langle|U(q)|^{2}\right\rangle$ có thể xác định bằng quy tắc Matthiessen

$$
\left\langle|U(q)|^{2}\right\rangle=\sum_{i}\left\langle\left|U_{i}(q)\right|^{2}\right\rangle
$$

trong đó, $U_{i}(q)=\int_{-\infty}^{+\infty} d z|\psi(z)|^{2} U_{i}(q, z)$ là ảnh Fourier của thế tán xạ thứ $i$ (chưa tính tới hiệu ứng chắn) với trọng là mật độ xác suất $|\psi(z)|^{2}$ của hạt tải cho bởi phương trình $(1)$ và $\left\langle\left|U_{i}(q)\right|^{2}\right\rangle$ là $\mathrm{ACF}$ của nó. Dạng cụ thể của $\mathrm{ACF}$ cho từng cơ chế tán xạ được giới thiệu trong phần 2.3.

Khi đã biết thời gian hồi phục trung bình, độ linh động của $2 \mathrm{DEG}$ có thể tìm từ công thức

$$
\mu_{0}=e\langle\tau\rangle / m^{*} \text {. }
$$

Vì chưa có lý thuyết cho LFC có tính tới tương tác giữa hai loại spin lên/xuống nên chỉ hai trường hợp được nghiên cứu là không phân cực $B=0$, $\langle\tau\rangle=\left\langle\tau_{+}\right\rangle=\left\langle\tau_{-}\right\rangle$và phân cực coi như hoàn toàn khi $B>B_{\mathrm{s}},\langle\tau\rangle=\left\langle\tau_{+}\right\rangle,\left\langle\tau_{-}\right\rangle=0$.

\subsection{Các cơ chế tán xạ được khảo sát}

Hai cơ chế tán xạ được nghiên cứu là RIS và IRS, cơ chế tán xạ RIS xuất hiện do một mặt phẳng các tạp chất mang điện có mật độ $N_{i}$ hình thành cách giao diện $\mathrm{SiGe/Si} \mathrm{một} \mathrm{khoảng} \mathrm{cách} z_{i}$ được minh họa trong Gold (1987), theo đó ACF của cơ chế này có dạng

$$
\left\langle\left|U_{1}(q)\right|^{2}\right\rangle=N_{i}\left(\frac{2 \pi e^{2}}{\in_{L} q}\right)^{2} F_{R}\left(q, z_{i}\right)^{2}
$$

với $N_{i}$ là mật độ tạp chất mang điện trên mặt phẳng $z_{i}$ và 


$$
F_{R}\left(q, z_{i}\right)=\frac{8 \pi^{2}}{a q} \frac{1}{4 \pi^{2}+a^{2} q^{2}}\left\{\begin{array}{l}
\frac{1}{2} e^{q z_{i}}\left(1-e^{-a q}\right), z_{i}<0 \\
1-\frac{1}{2} e^{-q z_{i}}-\frac{1}{2} e^{-q\left(a-z_{i}\right)}+\frac{a^{2} q^{2}}{2 \pi^{2}} \sin ^{2}\left(\frac{\pi z_{i}}{a}\right), 0 \leq z_{i} \leq a \\
\frac{1}{2} e^{-q\left(a-z_{i}\right)}\left(1-e^{-a q}\right), z_{i}>a
\end{array}\right.
$$

là thừa số dạng của thế tán xạ RIS lên $2 \mathrm{DEG}$ trong QW được khảo sát.

ACF của cơ chế IRS cho bởi (Gold, 1987)

$$
\left\langle\left|U_{2}(q)\right|^{2}\right\rangle=2\left(\frac{4 \pi}{a^{2}}\right)\left(\frac{m^{*}}{m_{z}}\right)^{2}\left(\frac{\pi}{k_{F} a}\right)^{4}\left(\varepsilon_{F} \Delta \Lambda\right)^{2} e^{-q^{2} \Lambda^{2} / 4}
$$

trong đó $\Delta$ biểu diễn độ nhám trung bình của giao diện và $\Lambda$ biểu diễn tham số độ dài tương quan của nhám trong mặt phẳng $x y$ của $2 \mathrm{DEG}$.

Ngoài ra, tương tác phonon-hạt tải được bỏ qua vì không đáng kể trong khoảng nhiệt độ khảo sát (Walukiewicz et al., 1984).

\subsection{Mật độ tới hạn}

Các kết luận trong Gold $(1997,2011)$ đã chỉ ra rằng MSE là cần thiết để mô tả được MIT ở mật độ thấp. Gọi $n_{\mathrm{MIT}}$ là mật độ ở đó xảy ra sự chuyển pha (mật độ tới hạn) và $\mu$ là độ linh động của $2 \mathrm{DEG}$ có tính tới MSE, theo Gold (1986, 2010), khi $n \geq n_{\mathrm{MIT}}$, độ linh động có thể được viết thành

$$
\mu=\mu_{0}(1-A),(A \leq 1),
$$

trong đó, $\mu_{0}$ xác định bằng phương trình (11). Tham số $A$ mô tả tác dụng của MSE, nó phụ thuộc vào thế tán $x a ̣$, hiệu ứng chắn và hệ số nén của $2 \mathrm{DEG}$ như sau:

$$
A=\frac{1}{2 \pi n^{2}} \int_{0}^{\infty} d q q \frac{\left\langle|U(q)|^{2}\right\rangle \Pi^{2}(q)}{\in_{q}^{2}} .
$$

Mật độ tới hạn $n_{\mathrm{MIT}}$ được tìm từ việc giải phương trình $A=1$. Khi $n<n_{\text {MIT }}$, độ linh động $\mu$ bằng 0 .

\section{KẾT QUẢ VÀ THẢO LUẬN}

\subsection{Sự phụ thuộc của độ linh động vào nhiệt độ và từ trường dưới tác dụng của RIS}

Các tính toán trong phần này sử dụng các giá trị tham số $a=200 \AA, z_{i}=-125 \AA, N_{i}=0,92 \times 10^{11} \mathrm{~cm}^{-}$ ${ }^{2}, \Delta=6 \AA, \Lambda=30 \AA$ được lấy từ Gold (2010).
Hình 1 biểu diễn kết quả tính toán tại các nhiệt độ khác nhau cho độ linh động $\mu$ của $2 \mathrm{DEG}$ với cơ chế tán xạ RIS khi không có mặt từ trường $\left(B=0, g_{s}=2\right)$. Các nốt tròn là dữ liệu từ thực nghiệm trong Wilamowski et al. (2001) tại 0,36 K. Kết quả nghiên cứu cho thấy rằng độ linh động tại các nhiệt độ dưới $2 \mathrm{~K}$ rất gần với độ linh động tại 0 $\mathrm{K}$ với sai lệch không quá $4,4 \%$. Do đó, dữ liệu từ thực nghiệm hoàn toàn có thể sử dụng để so sánh với độ linh động được tính toán tại $0 \mathrm{~K}$ với độ chính xác cao, điều này cho thấy kết quả của Gold (2010) rất đáng tin cậy.

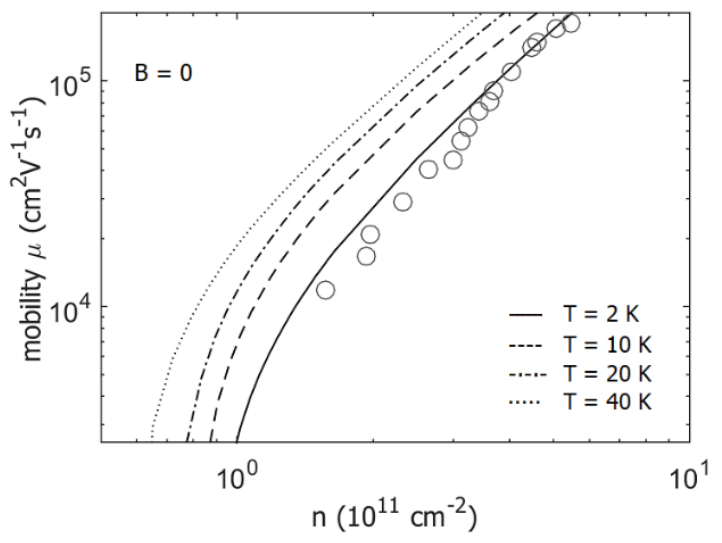

Hình 1. Biểu diễn độ linh động theo mật độ hạt tải tại các nhiệt độ khác nhau khi không có mặt từ trường. Các nốt tròn biểu diễn số liệu thực nghiệm từ Wilamowski et al. (2001)

Hình 1 cho thấy sự thay đổi rõ rệt của mật độ tới hạn $n_{\mathrm{MIT}}$ theo nhiệt độ (các giao điểm của đồ thị và trục hoành). Hình 2 cung cấp một cái nhìn chi tiết hơn về sự phụ thuộc này. Đối với mẫu $\mathrm{QW}$ này, tác dụng của nhiệt độ lên MIT là đáng kể ở các nhiệt độ trên $2 \mathrm{~K}$. Cụ thể MIT sẽ xuất hiện ở các mật độ hạt tải bé hơn khi tăng nhiệt độ của hệ bởi vì sự gia tăng ảnh hưởng của dao động nhiệt. Vấn đề này được khảo sát kĩ hơn trong phần 3.2.

Khi phân cực hệ khí bằng một từ trường có giá trị bão hòa $B_{s}$, MIT xuất hiện tại các mật độ tới hạn cao hơn so với khi không có từ trường (Hình 3 ). Như 
vậy, từ trường có thể làm hệ chuyển pha từ "dẫn điện" sang "cách điện", đặc điểm này có thể tạo tiền đề cho các ứng dụng vi điện tử chẳng hạn công tắc ngắt mạch bằng từ trường.

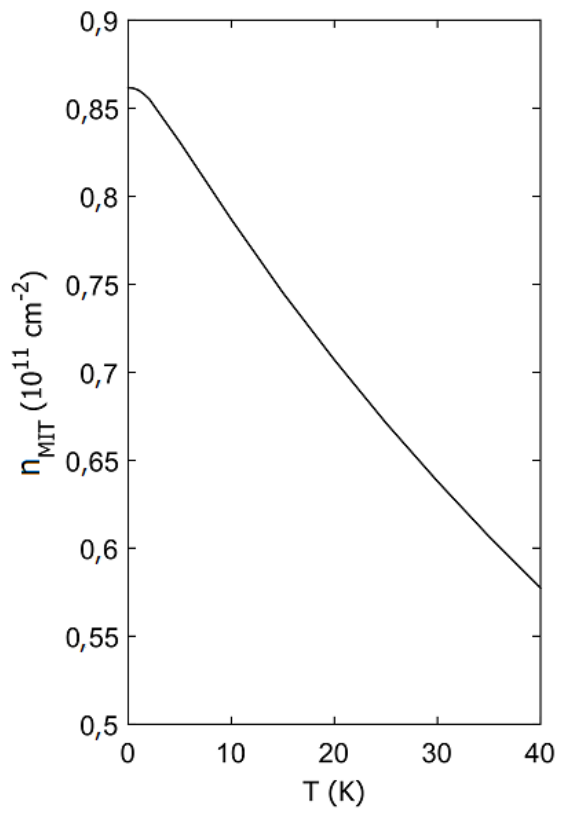

Hình 2. Biểu diễn mật độ tới hạn theo các nhiệt độ khác nhau khi $B=0$

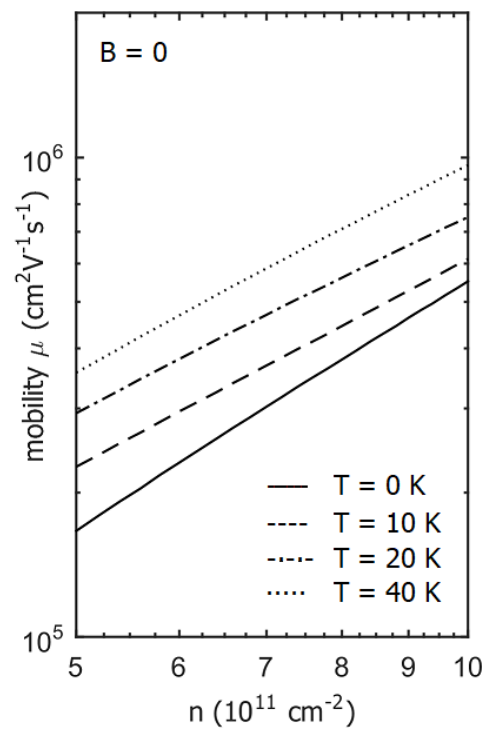

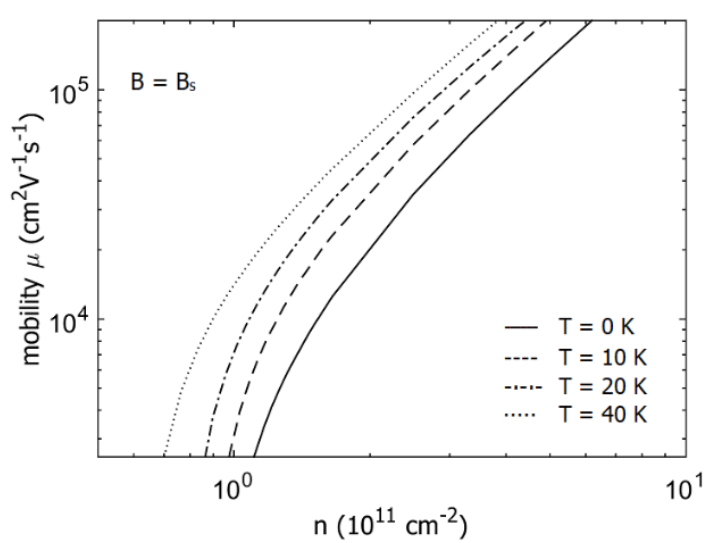

Hình 3. Biểu diễn độ linh động theo mật độ hạt tải tại các nhiệt độ khác nhau trong từ trường $B=B$ s

Để quan sát chi tiết hơn tác dụng của nhiệt độ và từ trường lên $2 \mathrm{DEG}$, tính toán độ linh động trong vùng mật độ cao $5 \times 10^{11} \mathrm{~cm}^{-2}$ tới $10 \times 10^{11} \mathrm{~cm}^{-2}$ được thực hiên. Kết quả được biểu diễn trong Hình 4 (phải) cho thấy từ trường có tác dụng làm giảm độ linh động trên mọi giá trị nhiệt độ được khảo sát. Chú ý rằng thang biểu diễn của đồ thị là $\log$ cho cả 2 trục, như vậy độ linh động ở trong vùng mật độ này có dáng điệu tuyến tính. Kết quả cũng cho thấy trong vùng mật độ này (Hình 4) và các mật độ thấp hơn (Hình 1 và Hình 3), một khi hệ ở trong pha "dẫn điện", độ linh động sẽ tăng theo nhiệt độ.

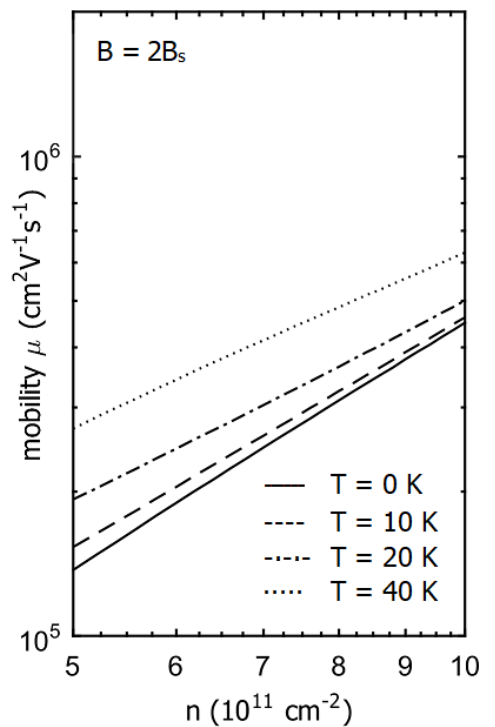

Hình 4. Biểu diễn độ linh động theo mật độ hạt tải tại các nhiệt độ khác nhau khi $B=0$ (trái) và $B=$ $2 B_{\mathrm{s}}$ (phải) cho trường hợp RIS 


\subsection{Sự phụ thuộc của mật độ chuyển pha vào nhiệt độ và khoảng cách lớp tạp chất}

Trong phần này, sự phụ thuộc của mật độ tới hạn vào nhiệt độ cho cơ chế RIS theo khoảng cách $z_{i}$ của lớp tạp chất được khảo sát. Các tham số được giữ nguyên như phần 3.1 tuy nhiên $z_{i}$ sẽ nhận các giá trị $a / 2,0,-a / 2$ và $-a$ với $a$ là bề rộng $\mathrm{QW}$. Hình 5 (trái) cho thấy khi không có từ trường, với các giá trị nhiệt độ dưới $5 \mathrm{~K}$, có thể xem mật độ chuyển pha hầu như không thay đổi. Trên $5 \mathrm{~K}$, mật độ tới hạn giảm đơn điệu theo nhiệt độ. Tuy nhiên, khi 2DEG bị phân cực mạnh như thể hiện trong Hình 5 (phải), sự thay đổi của mật độ tới hạn theo nhiệt độ không còn đơn điệu mà có đi qua một cực đại.

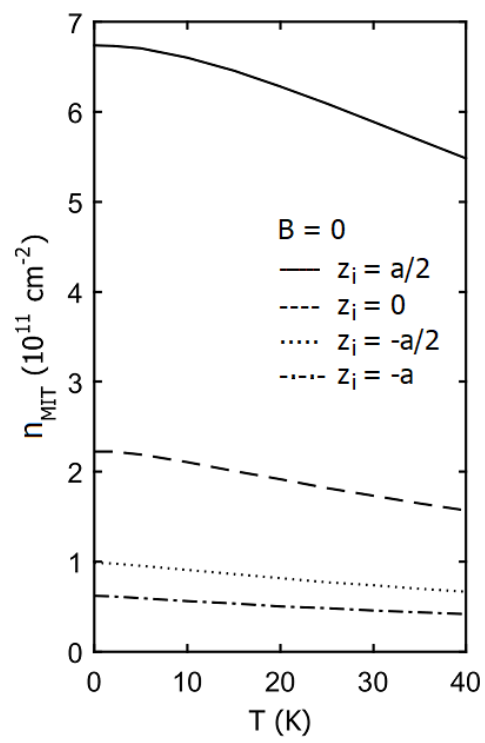

Trong cả hai trường hợp trên, lớp tạp chất càng nằm gần $\mathrm{QW}\left(z_{i}=0\right.$ - trên bề mặt và $z_{i}=a / 2$ - bên trong $\mathrm{QW}$ ) thì ảnh hưởng của nhiệt độ lên mật độ tới hạn càng rõ rệt. Nhìn chung khi tạp chất nằm xa QW, nó làm MIT xảy ra ở mật độ thấp hơn và ít phụ thuộc vào nhiệt độ hơn. Cụ thể, trong Hình 5 , đường biểu diễn ứng với 2 trường hợp $z_{i}=-a$ và $z_{i}=-a / 2$ nằm rất thấp và có dáng điệu phẳng hơn so với hai trường hợp $z_{i}=0$ và $z_{i}=a / 2$. Điều này có thể hàm ý rằng các tán xạ ngẫu nhiên tầm xa đã tăng cường tính định xứ của điện tử ở dưới đáy của dải con, khiến chúng ít nhạy cảm với nhiệt độ.

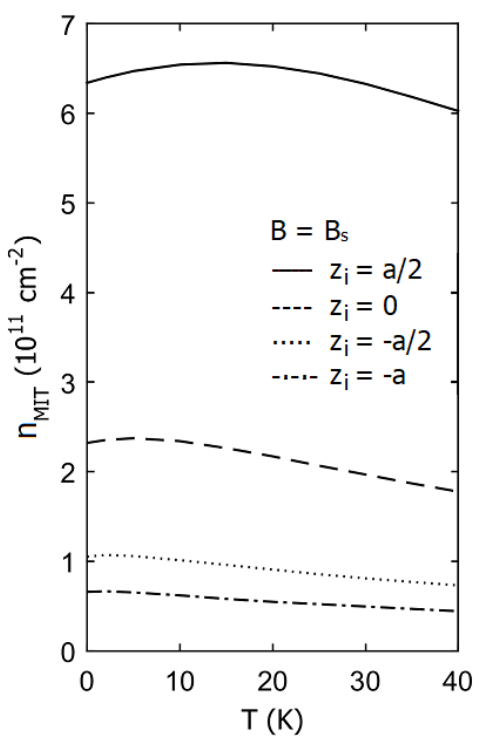

Hình 5. Biểu diễn mật độ tới hạn theo nhiệt độ tại các khoảng cách tạp chất khác nhau cho RIS khi $B$ $=0$ (trái) và $B=B_{\mathrm{s}}$ (phải)

\subsection{Tác dụng của bề rộng $\mathrm{QW}$ và từ trường lên mật độ chuyển pha do IRS}

Trong phần này, sự phụ thuộc của mật độ tới hạn vào bề rộng $\mathrm{QW}$ và nhiệt độ cho trường hợp của cơ chế IRS được nghiên cứu. Để nhất quán với Gold (2010), các tham số ở phần 3.1 được sử dụng, chỉ thay đồi bề rộng $a$ của QW.

Khanh and Quan (2013) đã khảo sát mật độ tới hạn theo bề rộng $a$ của QW chỉ cho cơ chế tán xạ RIS và thấy rằng khi tăng bề rộng $a$ từ $20 \AA ̊$ tới 200 Å, mật độ tới hạn $n_{\mathrm{MIT}}$ giảm đi. Nói cách khác chuyển pha sẽ xảy ra ở các mật độ hạt tải thấp trong những QW rộng. Kết quả tính toán cũng cho thấy hiện tượng tương tự cho cơ chế IRS (Hình 6). Tuy nhiên, dáng điệu đồ thị trong Hình 6 dốc xuống nhanh hơn, nghĩa là đóng góp của IRS vào $n_{\mathrm{MIT}}$ giảm nhanh hơn so với RIS khi tăng bề rộng $\mathrm{QW}$. Cụ thể, khi $a>70 \AA$, chỉ có cơ chế RIS tham gia chủ yếu vào hình thành MIT. Với những $a<30 \AA$, đóng góp của IRS mới trở nên trội hơn RIS. Điều này củng cố nhận định của Gold (2010) cho rằng những QW hẹp rất phù hợp để nghiên cứu MIT gây ra do cơ chế IRS. Kết quả này cũng giải thích vì sao IRS hầu như không tham gia vào cơ chế hình thành MIT trong thực nghiệm của Wilamowski et al. (2001) vì bề dày $\mathrm{QW}$ của mẫu lên tới $200 \AA$ trong khi đóng góp của IRS lên MIT chỉ đáng kể dưới $70 \AA$ tại $0 \mathrm{~K}$.

Đáng chú ý, tác dụng của IRS lên MIT càng bị dập đi nhanh chóng khi có sự tham gia của nhiệt độ. Trong miền bề rộng được khảo sát, từ 20 Å đến 100 
Å, tại $0 \mathrm{~K}$, giá trị $n_{\mathrm{MIT}}$ do IRS gây ra chỉ giảm từ từ (đường nét liền trong Hình 6). Trong khi đó, với sự có mặt của nhiệt độ, đồ thị $n_{\mathrm{MIT}}$ trở nên dốc đứng (các đường nét đứt) khi gặp một giá trị gọi là bề rộng tới hạn. Cụ thể, tại $T$ lần lượt là $40 \mathrm{~K}, 20 \mathrm{~K}, 10 \mathrm{~K}$, $5 \mathrm{~K}$ và $2 \mathrm{~K}$, đóng góp của IRS vào MIT sẽ bị dập tắt tương ứng tại các bề rộng tới hạn $36,40 \AA$, 41,8 $\AA$, $47,2 \AA, 53,2 \AA$ và $62.2 \AA$. Nhiệt độ càng cao, bề rộng tới hạn càng nhỏ. Tại $a=20 \AA$, tác dụng của RIS lên mật độ tới hạn chỉ chiếm $0,0750 \%$ so với IRS.

Vì QW 200 Å được xem là rộng nên các kết quả ở mục 3.1 và 3.2 tuy chỉ xét riêng RIS nhưng vẫn đảm bảo khi thêm IRS vào các cơ chế tán xạ.

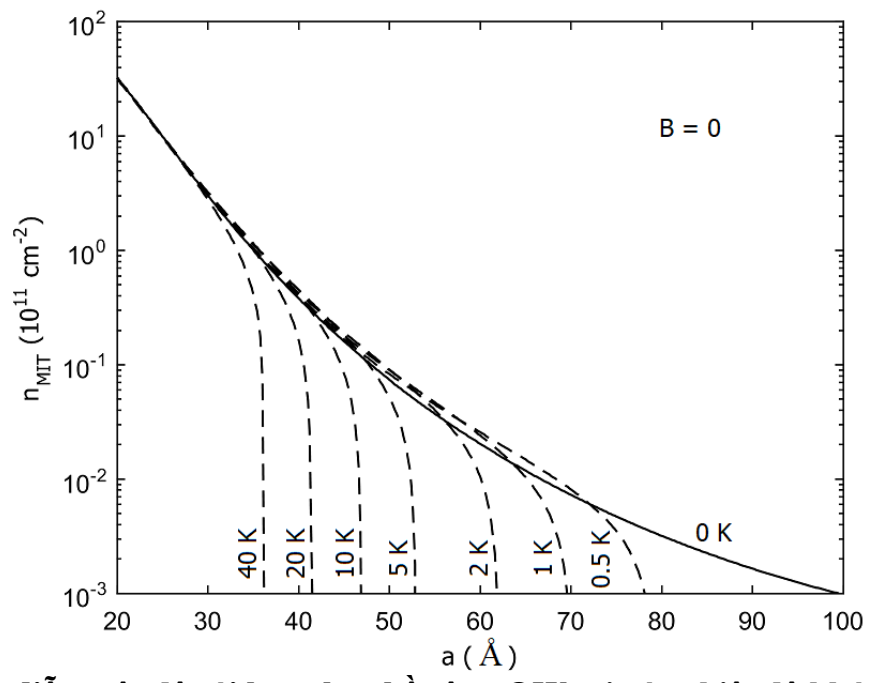

Hình 6. Biểu diễn mật độ tới hạn theo bề rộng $Q W$ tại các nhiệt độ khác nhau khi $B=0$

Bề rộng tới hạn khi $2 \mathrm{DEG}$ bị phân cực bằng từ trường $B=B_{s}$ cũng được khảo sát. Hình 7 cho thấy từ trường có tác dụng nâng nhẹ giá trị mật độ tới hạn $n_{\mathrm{MIT}}$ trên mọi bề rộng $\mathrm{QW}$ và tác dụng dập tắt ảnh hưởng của IRS lên MIT của nhiệt độ sẽ xảy ra tại các bề rộng tới hạn lớn hơn một chút. Với các mức nhiệt độ lần lượt là $40 \mathrm{~K}, 20 \mathrm{~K}, 10 \mathrm{~K}, 5 \mathrm{~K}, 2 \mathrm{~K}$, giá trị các bề rộng tới hạn là $37 \AA, 42 \AA, 47,4 \AA ⿻, 53,4 \AA$ và $62,4 \AA$.

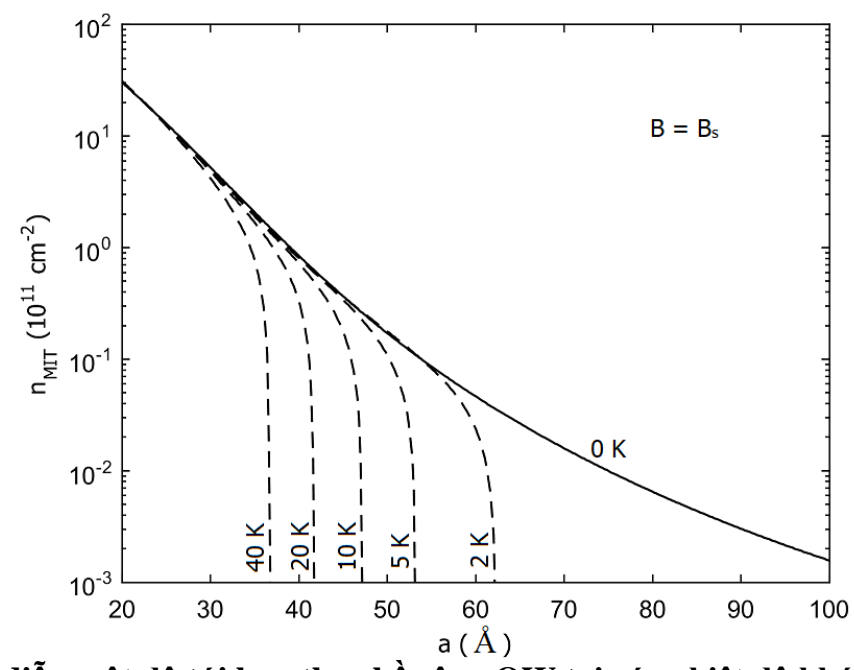

Hình 7. Biểu diễn mật độ tới hạn theo bề rộng $Q W$ tại các nhiệt độ khác nhau khi $B=B_{s}$

Các tính toán trong nghiên cứu này giả sử khối lượng hiệu dụng của $2 \mathrm{DEG}$ không phụ thuộc vào mật độ điện tử. Trong các cấu trúc Silic MOSFET với độ linh động rất cao, khối lượng hiệu dụng có thể không còn là hằng số, đặc biệt quanh mật độ tới hạn (Kravchenko \& Sarachik, 2004), điều này cần 
được thực nghiệm kiểm tra lại. Hơn nữa, giá trị từ trường bão hòa sử dụng trong nghiên cứu vốn được định nghĩa cho hệ không tương tác và chưa tính tới nhiệt độ. Tuy nhiên, Khanh (2011) đã chỉ ra rằng các kết quả dựa trên từ trường bão hòa này vẫn có giá trị ít nhất cho các mật độ đủ cao hoặc/và nhiệt độ đủ thấp.

\section{KẾT LUẬN}

Kết quả nghiên cứu cho thấy nhiệt độ của hệ khí trong thí nghiệm của Wilamowski et al. (2001) có thể xem là đủ thấp để xác nhận kết quả lý thuyết của Gold (2010) ở $0 \mathrm{~K}$, đồng thời sự gia tăng của độ linh động theo mật độ hạt tải và nhiệt độ trong 2DEG khi có hoặc không bị phân cực bởi từ trường được khảo sát. Nghiên cứu này cũng cho thấy sự phụ thuộc không thể bỏ qua của mật độ tới hạn vào nhiệt độ, khoảng cách pha tạp và bề rộng $\mathrm{QW}$ cho hai cơ chế tán xạ RIS và IRS. Các kết quả của nghiên cứu cung cấp gợi ý cho việc nuôi cấy mẫu và kiểm soát nhiệt độ khi đo đạc thực nghiệm và sẽ được củng cố khi các số liệu mới được ghi nhận.

\section{LÒ̀I CẢM TA}

Chúng tôi muốn bày tỏ lòng biết ơn đối với Trường Đại học Kiên Giang đã tài trợ tài chính trong suốt quá trình nghiên cứu này. Chúng tôi xin cảm ơn các vị phản biện và quý Tạp chí vì sự hỗ trợ mà chúng tôi nhận được.

\section{TÀI LIẸU THAM KHẢO}

Das Sarma, S., \& Hwang, E. H. (2005). Low-density spin-polarized transport in two-dimensional semiconductor structures: Temperaturedependent magneto resistance of Si MOSFETs in an in-plane applied magnetic field. Physical Review B, 72(20), 205303.

Gold, A., \& Götze, W. (1986). Localization and screening anomalies in two-dimensional systems. Physical Review B, 33(4), 2495-2511.

Gold, A. (1987). Electronic transport properties of a two-dimensional electron gas in a silicon quantum-well structure at low temperature. Physical Review B, 35(2), 723-733.

Gold, A., \& Calmels, L. (1993). Correlation in Fermi liquids: Analytical results for the local-field correction in two and three dimensions. Physical Review B, 48(16), 11622-11637.

Gold, A. (1994). Local-field correction for the electron gas: Effects of the valley degeneracy. Physical Review B, 50(7), 4297-4305.

Gold, A. (1997). The local-field correction for the interacting electron gas: many-body effects for unpolarized and polarized electrons. Zeitschrift für Physik B, 103(3), 491-500.

Gold, A. (2010). Mobility and metal-insulator transition of the two-dimensional electron gas in $\mathrm{SiGe} / \mathrm{Si} / \mathrm{SiGe}$ quantum wells. Journal of Applied Physics, 108(6), 063710.

Gold, A. (2011). Metal-insulator transition in $\mathrm{Si} / \mathrm{SiGe}$ heterostructures: mobility, spin polarization and Dingle temperature. Semiconductor Science and Technology, 26(4), 045017.

Khanh, N. Q. (2011). Transport properties of a spinpolarized quasi-two-dimensional electron gas in an $\mathrm{InP} / \mathrm{In}_{1-\mathrm{x}} \mathrm{Ga} \mathrm{a}_{\mathrm{A}} \mathrm{As} / \mathrm{InP}$ quantum well including temperature effects. Physica E, 43(9), 1712-1716.

Khanh, N. Q., \& Quan, N. M. (2013). Transport properties of a quasi-two-dimensional electron gas in a $\mathrm{SiGe} / \mathrm{Si} / \mathrm{SiGe}$ quantum well including temperature and magnetic field effects. Superlattices and Microstructures, 64, 245-250.

Kravchenko, S. V., \& Sarachik, M. P. (2004). Metalinsulator transition in two-dimensional electron systems. Reports on Progress in Physics, 67(1), $1-44$.

Laikhtman, B., \& Kiehl, R. A. (1993). Theoretical hole mobility in a narrow $\mathrm{Si} / \mathrm{SiGe}$ quantum well. Physical Review B, 47(16), 10515.

Walukiewicz, W., Ruda, H. E., Lagowski, J., \& Gatos, H. C. (1984). Electron mobility in modulation-doped heterostructures. Physical Review B, 30(8), 4571-4582.

Wilamowski, Z., Sandersfeld, N., Jantsch, W., Többen, D., \& Schäfffler, F. (2001). Screening breakdown on the route toward the metalinsulator transition in modulation doped $\mathrm{Si} / \mathrm{SiGe}$ quantum wells. Physical Review Letters, 87(2), 026401.

Zeller, Ch., \& Abstreiter, G. (1986). Electric subbands in $\mathrm{Si} / \mathrm{SiGe}$ strained layer superlattices. Zeitschrift für Physik B, 64(2), 137-143. 